Memorias del VII Encuentro Nacional de Experiencias en la Enseñanza de la Biología y la Educación Ambiental y II Congreso Nacional de Investigación en la Enseñanza de la Biología

\title{
FORMACIÓN EN INVESTIGACIÓN EN FUTUROS PROFESORES DE BIOLOGÍA. VALIDACIÓN DE UN INSTRUMENTO PARA INDAGAR EXPERIENCIAS Y REFERENTES SOBRE INVESTIGACIÓN
}

\section{FORMATION IN INVESTIGATION IN FUTURE TEACHERS OF BIOLOGY. VALIDATION OF AN INSTRUMENT TO INVESTIGATE EXPERIENCES AND POINTS OF REFERENCE ABOUT RESEARCH}

Édgar Orlay Valbuena Ussa ${ }^{1}$

Julio Alejandro Castro Moreno ${ }^{2}$

Análida Altagracia Hernández Pichardo ${ }^{3}$

Yolanda Catalina Vallejo Ovalle ${ }^{4}$

Laura Marcela Trujillo Castro ${ }^{5}$

Lizeth Aixa Ariza Bareño ${ }^{6}$

\section{Resumen}

En este escrito presentamos un avance de la aplicación de uno de los instrumentos propuestos en la investigación, Formación en investigación en futuros profesores de Biología (financiado por el CIUP) ${ }^{7}$ cuyo propósito es contribuir a la caracterización de la formación en investigación en futuros profesores de Biología, al interior del Proyecto Curricular de Licenciatura en Biología (PCLB) de la Universidad Pedagógica Nacional (UPN). Para el abordaje de la investigación los referentes teóricos que se tienen en cuenta son: investigación y formación, investigación y formación de docentes de Ciencias Naturales y enseñanza de las ciencias.

La investigación se realiza desde la perspectiva cualitativa con enfoque interpretativo, donde los datos a recolectar se obtienen de informes de los futuros profesores de Biología, documentos del PCLB, y de las declaraciones de los sujetos implicados en los procesos formativos en investigación al interior del PCLB (directivos, formadores y futuros profesores). Por consiguiente, el análisis documental y de contenido, la conformación de grupos focales, la realización de entrevistas y cuestionarios, corresponden a técnicas de

\footnotetext{
${ }^{1}$ Director Departamento de Biología UPN - valbuena@pedagogica.edu.co

${ }^{2}$ Profesor Departamento de Biología UPN - alecasmor@yahoo.es

${ }^{3}$ Profesora Departamento de Biología UPN - ahernandez@pedagogica.edu.co

${ }^{4}$ Profesora Departamento de Biología UPN - yvallejo@pedagogica.edu.co

${ }^{5}$ Estudiante Licenciatura en Biología UPN - dbi_Itrujillo432@pedagogica.edu.co

${ }^{6}$ Estudiante Licenciatura en Biología UPN - dbi.lariza@pedagogica.edu.co

${ }^{7}$ Centro de Investigaciones de la UPN.
} 
Memorias del VII Encuentro Nacional de Experiencias en la Enseñanza de la Biología y la Educación Ambiental y II Congreso Nacional de Investigación en la Enseñanza de la Biología

recolección de información. Para el caso de la presente ponencia, se exponen los hallazgos parciales de la investigación, obtenidos a partir de la aplicación de un cuestionario a 23 futuros profesores de Biología quienes se encuentran cursando el espacio académico "Seminario de Investigación". De acuerdo con los datos arrojados por el cuestionario, se hace manifiesto que algunos futuros docentes han tenido cierta relación con la investigación durante su formación, a partir del desarrollo de proyectos transversales en cada semestre y durante la práctica pedagógica. Sin embargo dicho acercamiento se ha encontrado obstaculizado por algunas dinámicas del PCLB.

Palabras Clave: Investigación, formación docente, Conocimiento Profesional del Profesor de Ciencias, Enseñanza, cuestionario.

\section{Absctract}

In this writing we show an advance of the application of one of the instruments proposed in the investigation, Formation in investigation in future teachers of Biology which intention is to contribute to the characterization of the formation in investigation in future teachers of Biology, to the interior of the Project Curricular of Master in Biology (PCLB) of the Universidad Pedagógica Nacional. For the boarding of the investigation the theoretical concerning are, investigation and formation, investigation and teachers' formation of Natural Sciences and education of the sciences.

The investigation, is realized from the qualitative perspective by interpretive approach, where the information to gathering is obtained of, report of the future teachers of Biology and documents of the PCLB, from the declarations of the subjects involved in the formative processes in investigation to the interior of the PCLB (managerial, forming and future teachers). Consequently, documentary analysis and of content, focal groups, interview and questionnaires, they correspond to techniques of compilation of information. For the case of the present presentation, there are exposed the partial findings of the investigation obtained from the application of a questionnaire to 23 future teachers of Biology who are dealing the space of Seminar of investigation. In agreement to the information thrown by the questionnaire, it appears that some educational futures expound they have had certain relation with the investigation during their formation, from the development of transverse projects in every semester and during the pedagogic practice, nevertheless the above mentioned approximation has been prevented by the different dynamics of the PCLB. 
Memorias del VII Encuentro Nacional de Experiencias en la Enseñanza de la Biología y la Educación Ambiental y II Congreso Nacional de Investigación en la Enseñanza de la Biología

Key Words: Investigation, educational formation, Professional Knowledge of the Sciences' Teacher, teaching, questionnaire.

\section{Introducción}

La investigación en el marco del Proyecto Curricular de la Licenciatura en Biología (PCLB) se asume como, "un proceso formativo sistemático, en el cual se propende por el desarrollo de habilidades competencias que permiten al Licenciado el logro de los objetivos propuestos; por ello se prevé involucrar activamente al estudiante en actividades relacionadas con la Investigación : a) Pedagógica y Didáctica, a partir del conocimiento construido por la humanidad, que incluye también lo gestado en las investigaciones de las líneas del Departamento, pertinentes con el Proyecto Curricular, b) investigación en Biología y áreas afines" (PCLB,1998). Lo anterior, es evidente en los diferentes ambientes de formación, ciclos, ejes y espacios académicos.

Según Bondarenko (2009), citado por Valbuena et al (2013) la relación entre la formación docente y la investigación es un tema que aún se encuentra poco definido, escasamente estudiado y en consecuencia poco tenido en cuenta en los espacios académicos. La autora continua exponiendo que "esta situación se debe, en parte, al hecho de existir una falta de claridad pedagógica en las respuestas a las dos preguntas fundamentales: a) ¿qué significa ser docente? y b) ¿qué significa investigar? La ausencia de respuestas contundentes a estas preguntas hace aún más difícil entender la relación que debe existir entre ambas" (Bondarenko, 2009 p.1).

De acuerdo con esta autora, la relación entre investigación y formación docente en gran medida se presenta de manera abstracta, sin que ello implique modificación de estructuras académicas o prácticas pedagógicas. Sin embargo, a pesar de esta situación, "actualmente se está notando un creciente interés hacia este tema por parte de investigadores, profesores formadores y hasta los mismos estudiantes (futuros docentes), debido, principalmente, al cambio paradigmático que se está presentando en todos los ámbitos de la sociedad, cambio que se manifiesta a través de la ruptura de las relaciones tradicionales de poder, desvanecimiento del concepto de la verdad, cuestionamiento del método científico, caos conceptual y otros fenómenos afines" (Bondarenko, 2009 p. 2 ).

Formar en investigación no es asunto simple ni fácil, por el contrario es complejo y amerita ser objeto de investigación; en ese sentido se pretende 
Memorias del VII Encuentro Nacional de Experiencias en la Enseñanza de la Biología y la Educación Ambiental y II Congreso Nacional de Investigación en la Enseñanza de la Biología

abordar el PCLB de la Universidad Pedagógica Nacional (UPN), ya que en este se pretende la formación investigativa de los futuros profesores. Así, en la investigación que estamos proponiendo, buscamos sistematizar y analizar distintos procesos al interior del PCLB, los cuales pretenden formar en investigación a los futuros licenciados; para tal fin, seleccionaremos entre las actividades y espacios el Seminario de investigación, el cual es cursado por estudiantes del ciclo de profundización (séptimo semestre en adelante), quienes se encuentran realizando el proyecto de práctica o el anteproyecto de grado.

Pretendemos, entonces caracterizar y analizar procesos del PCLB que constituyen la investigación como componente formativo en los futuros profesores de Biología, lo cual implica asumir problemas como: en dichos procesos formativos ¿qué referentes teóricos y metodológicos de investigación se abordan?, ¿qué caracteriza los problemas de investigación?, ¿cuáles son los objetos de investigación?; además, en el PCLB: ¿qué elementos promueven la formación en investigación?, ¿qué elementos obstaculizan la formación en investigación?, ¿cómo se pueden mejorar los procesos de formación en investigación?, ¿de qué manera los futuros profesores se integran a los procesos investigativos del Departamento de Biología y de la UPN?. En suma, la investigación propuesta se puede resumir en la siguiente pregunta problema: ¿Qué caracteriza la formación en investigación, en futuros profesores de Biología al interior del PCLB de la Universidad Pedagógica Nacional?

En ese sentido, en la presente ponencia se exponen los hallazgos preliminares de la investigación obtenidos a partir de la validación y aplicación de un cuestionario (instrumento para recolección de información), mediante el cual se indagaron las experiencias y referentes sobre investigación en 23 futuros profesores de Biología, que se encuentran cursando el Seminario de investigación.

\section{Marco De Referencia}

\section{Investigación y Formación Docente}

La construcción y reconstrucción del conocimiento del profesor suele ubicarse desde lugares distintos a los que acontecen al mismo y a quienes lo constituyen, al respecto Porlán y Rivero (1998) señalan que “...se suele pensar con frecuencia que los profesores van a comprender, asimilar y hacer suyas las nuevas propuestas, dado que éstas son "racionales" y están avaladas por 
Memorias del VII Encuentro Nacional de Experiencias en la Enseñanza de la Biología y la Educación Ambiental y II Congreso Nacional de Investigación en la Enseñanza de la Biología

algún tipo de legitimidad científica... los investigadores suelen pensar que sus evidencias y hallazgos son entendidos como tales por los profesores y que, por lo tanto, puede darse una transferencia directa entre investigación didáctica y actividad docente en beneficio de la mejora de la enseñanza. Mientras los investigadores discuten sobre la pertinencia de sus propuestas, esperando que el profesor las asuma, éstos, ajenos a la polémica, siguen trabajando mayoritariamente desde los enfoques más tradicionales" (Porlán \& Rivero, 1998 pp. 8 - 9).

En consecuencia, se requiere hacer partícipes a los profesores de la investigación de su práctica, para lo cual es fundamental empezar su proceso de formación en investigación desde sus estudios de licenciatura. Al respecto, Roa afirma que "el profesor entra a la vida del mundo de la educación formal, desde muy temprana edad, y empieza a construir concepciones sobre el conocimiento, la enseñanza y aprendizaje desde referentes prácticos y los valida en forma empírica. Su relación con el conocimiento es, en parte, la que desarrolla su profesión a través de la enseñanza, es la manera como concibe que el conocimiento se ha construido y se construye, se aprende. Su enseñanza está cargada de variados esquemas que orientan sus actitudes, decisiones, rutinas, creencias que posibilitan y crean formas de aprendizaje" (2011 p. 50). Al respecto, si bien es cierto que los futuros profesores tienen ya construidos conocimientos sobre la enseñanza, el aprendizaje, la evaluación y el currículo, entre otros, estos no deberían ser los que prevalezcan ni orienten su acción profesional; se requiere entonces construir un conocimiento profesional particular, para lo cual la investigación constituye un elemento fundamental.

\section{Investigación y formación de docentes de ciencias naturales}

Valeiras (2005) plantea que cuando se piensa sobre el rol que tiene la investigación en la formación del docente de ciencias, resulta primordial establecer acuerdos acerca de lo que significa investigar, siendo importante diferenciar entre la investigación del área disciplinar y la investigación en educación en ciencias. Lo anterior resulta relevante, dado que en algunas instituciones formadoras de docentes todavía se está impartiendo un estilo de formación en investigación basado en parámetros de la ciencia positivista, colaborando de esta forma a construir un determinado modelo de investigación en los docentes, permeando así los desarrollos de: las prácticas de aula, los seminarios temáticos y los trabajos prácticos que llevan a cabo algunas materias (Valeiras, 2005). 
Memorias del VII Encuentro Nacional de Experiencias en la Enseñanza de la Biología y la Educación Ambiental y II Congreso Nacional de Investigación en la Enseñanza de la Biología

Según el mismo autor, el modelo de ciencia que poseen los docentes en formación incide al momento de elaborar propuestas de investigación de aula, ya que dichas propuestas ponen en juego sus concepciones epistemológicas y didácticas, creencias y teorías personales, las cuales en gran parte están influenciadas por los procesos de formación, estableciéndose imaginarios sobre la enseñanza de las ciencias, los cuales están vinculadas con lo que para ellos significa investigar. Por otra parte, incorporar la investigación a la formación docente resulta primordial debido a que favorece la figura del profesor ya sea por lo que significa para su rol profesional, como por los aportes que hace sobre al conocimiento de la enseñanza y del aprendizaje (Valeiras, 2005).

\section{Metodología}

La investigación se propone desde la perspectiva de investigación cualitativa, la cual, según Carrasco y Caldero (2000) y Pérez (1998) (Citado por Valbuena et al, 2013), se caracteriza porque intenta acercarse al máximo a los sujetos buscando una "inmersión" en las situaciones; no busca la generalización sino estudiar singularidades en profundidad, intenta estudiar los sujetos como un todo en el contexto de su pasado y de las situaciones en las que se hallan; intenta describir e interpretar las situaciones, significados, intenciones, creencias, motivaciones, expectativas y otras características que no son observables directamente ni son susceptibles de experimentación o cuantificación, el enfoque empleado es el interpretativo.

Para obtener los datos se acudió al cuestionario, el que según Korman (1986) citado por Valbuena et al (2013), es concebido como "una reunión de un grupo de individuos seleccionados por los investigadores para discutir y elaborar, desde la experiencia personal, una temática o hecho social que es objeto de investigación" (Valbuena et al, 2013), en este caso: la formación en investigación.

El instrumento que se presenta como resultado de investigación, fue implementado en un grupo de 23 futuros profesores del PCLB, quienes cursan el componente Seminario de Investigación. Dichos estudiantes se encuentran en diferentes niveles del ciclo de profundización ${ }^{8}$, es decir que en el seminario convergen estudiantes que culminaron el ciclo de fundamentación (de séptimo

\footnotetext{
${ }^{8}$ Nivel formativo en el marco de la estructura curricular de la Licenciatura en Biología de la UPN.
} 
Memorias del VII Encuentro Nacional de Experiencias en la Enseñanza de la Biología y la Educación Ambiental y II Congreso Nacional de Investigación en la Enseñanza de la Biología

semestre en adelante), donde algunos están desarrollando la práctica pedagógica y otros realizan el anteproyecto de trabajo de grado.

Los datos obtenidos en este primer acercamiento, provienen del cuestionario propuesto (con previo consentimiento informado de los 23 participantes), el cual arrojó como resultado las declaraciones escritas de los futuros profesores, en una de las sesiones de este; quienes para dar respuestas utilizaron un seudónimo. Este instrumento permitió el acercamiento a las concepciones que poseen acerca de la investigación, así como indagarlas y analizarlas.

Los datos obtenidos en este primer acercamiento provienen del cuestionario propuesto por el grupo del proyecto de investigación: Formación en investigación en futuros profesores de Biología, quienes validaron el instrumento mediante las respuestas de una prueba piloto, la cual consistió en emplear el cuestionario con algunos estudiantes del PCLB que no pertenecen al grupo de estudio. Posterior a la validación, el instrumento fue aplicado a 23 futuros profesores de Biología en una sesión del Seminario de Investigación, con previo consentimiento informado. Algunos estudiantes utilizaron su nombre y otros un seudónimo, ellos realizaron declaraciones escritas con respecto a las experiencias y referentes sobre investigación.

La sistematización de los datos obtenidos a partir de las declaraciones se realizó teniendo en cuenta el método de análisis de contenido (Bardín, 1977:)

$\checkmark$ Extracción de las unidades de información con sentido partir de cada una de las fuentes.

$\checkmark$ Agrupación de las unidades de información en las diferentes categorías de investigación.

$\checkmark$ Análisis de las proposiciones (en este caso, en relación con las actividades formativas).

\section{Resultados Y Discusión}

Presentamos los resultados en tres momentos, los cuales se desarrollaron cronológicamente. En primera instancia, la estructuración del cuestionario, que permitió la aplicación del mismo, el cual posibilitó la sistematización de la información, donde se presentan los hallazgos parciales de la investigación.

\section{Estructuración del cuestionario}


Memorias del VII Encuentro Nacional de Experiencias en la Enseñanza de la Biología y la Educación Ambiental y II Congreso Nacional de Investigación en la Enseñanza de la Biología

El instrumento tenía como propósito aportar elementos para la caracterización y comprensión de la formación en investigación en futuros profesores del PCLB. Para la estructuración del cuestionario se realizaron reuniones por parte del equipo investigador, en donde se discutió en torno a las preguntas que serían la base del instrumento. Así mismo, cada uno de investigadores realizó un ejercicio de validación de dichas preguntas con estudiantes de similares características a la población objeto de estudio, posteriormente, y a partir del ejercicio de validación, se estructuró la versión final del instrumento que planteó las siguientes preguntas:

¿Durante su experiencia como estudiante del Proyecto Curricular de Licenciatura en Biología ha tenido alguna relación con la investigación? Si su respuesta es afirmativa, por favor relate de la manera más precisa cómo ha sido. Para tal fin, en su relato le solicitamos incluir además de los aspectos que usted considere relevantes, los siguientes: ¿en qué espacios académicos o actividades ha tenido relación con la investigación?, las finalidades y problemas abordados en las investigaciones (aproximaciones a investigaciones) realizadas, y relación de dicha experiencia en investigación con la formación como futuro (a) profesor (a) de Biología. Si su respuesta es negativa, argumente de la manera más precisa ¿qué ha obstaculizado su relación con la investigación durante su proceso de formación?

\section{Aplicación del cuestionario}

El instrumento se aplicó a 23 futuros profesores de Biología durante una sesión del Seminario de Investigación (espacio académico objeto de la presente investigación). Para ello, se realizó la presentación de la investigación, seguido de la aprobación por parte de los estudiantes para participar en la misma. Posteriormente, cada uno recibió el instrumento impreso para dar respuesta al planteamiento que allí se presenta. Igualmente, algunos estudiantes utilizaron su nombre y otros un seudónimo.

\section{Sistematización de los datos obtenidos}

Después de la aplicación del instrumento, se lograron obtener diferentes unidades de información que se encontraron en los resultados, las cuales nos sirvieron para agruparlas en diferentes categorías que corresponden a las preguntas realizadas en el cuestionario. 
Memorias del VII Encuentro Nacional de Experiencias en la Enseñanza de la Biología y la Educación Ambiental y II Congreso Nacional de Investigación en la Enseñanza de la Biología

A continuación mostraremos las preguntas realizadas, y a partir de ellas las categorías obtenidas, igualmente se exponen algunas unidades de información que sustentan las categorías, para ello las citas correspondientes se hacen con un número designado en la sistematización y el año en el que se realizó el cuestionario.

1. ¿Durante su experiencia como estudiante del Proyecto Curricular de Licenciatura en Biología ha tenido alguna relación con la investigación?

A partir de la sistematización de las respuestas a esta pregunta se construyeron tres categorías. La primera corresponde a los estudiantes que consideran que sí han tenido alguna relación con la investigación, mostrando sus diferentes puntos de vista en cuanto a cómo ha sido este proceso. Donde en muchos casos los estudiantes afirman haber tenido relación con la investigación desde un primer momento, en muchos casos desde primer semestre, a través de los diferentes proyectos propuestos en cada eje curricular del PCLB, como lo muestran las siguientes unidades de información:

"en mi experiencia como estudiantes de la licenciatura en Biología si he tenido alguna relación con la investigación. En los proyectos de semestre hay un acercamiento al planteamiento de lo que es una investigación" $(2,2013)$

"pienso que si hemos tenido experiencia con la investigación desde el primer semestre. Por el manejo del proyecto transversal ya que se realiza acercamiento a la investigación" $(23,2013)$

Igualmente, en esta categoría algunos estudiantes afirman tener una aproximación con algunos aspectos correspondientes a los que se puede llamar investigación, e incluso otras personas muestran en sus respuestas que se han acercado a la investigación desde otros contextos, por ejemplo:

"realmente, si he tenido oportunidades de realizar Investigación, pero no ha sido en los espacios académicos de la Universidad; sino en otros contextos" $(17,2013)$

La segunda categoría corresponde a las personas que consideran que no han tenido ninguna relación con la investigación; donde las diferentes personas respondieron que ello es debido a que el desarrollo de proyectos en los diferentes semestres no son una verdadera investigación, sino que simplemente son trabajos de documentación sin una rigurosidad, añadiendo también que estos proyectos se desarrollan en un tiempo corto, aspecto que no se puede manejar en una investigación. Algunas de estas apreciaciones son las siguientes: 
Memorias del VII Encuentro Nacional de Experiencias en la Enseñanza de la Biología y la Educación Ambiental y II Congreso Nacional de Investigación en la Enseñanza de La Biología

"No he tenido relación con la investigación en todo el pregrado se manejan proyectos por semestre o por materias, pero considero que no son proyectos de investigación sino, que son más de relaciones o consultas y que no se les puede comparar en cuanto a lo que se entiende por investigación" $(6,2013)$

"durante mi experiencia en el proyecto curricular considero que no he tenido experiencia con la investigación como tal, sino mi experiencia ha sido la elaboración de proyectos por semestres o por materias" $(5,2013)$

Por otra parte, algunas de estas personas manifiestan no tener ninguna relación con este proceso, pues muchos no tienen claro qué es investigación, como se expresa en la siguiente unidad de información:

\section{"no he tenido ninguna relación con la investigación pues a lo largo de los semestres no se explica o no se tiene claro el concepto de investigar" $(3,2013)$}

La tercera y última categoría que emergió para esta pregunta se denomina Otros, donde algunas personas no responden, y otras muestran una relación confusa con la investigación, puesto que para ellas la investigación está planteada desde el campo social a nivel educativo, y no en otro campo.

"la relación con la investigación durante mi formación ha sido de cierta manera un tanto confusa, ya que el proyecto de la licenciatura está planeado con una formación investigativa en el campo social que nos compete que es la educación" $(8,2013)$

2. ¿En qué espacios académicos o actividades, ha tendido relación con la investigación?

En cuanto a las respuestas a esta pregunta, es evidente la mención que se hace de los diferentes espacios académicos o actividades en los cuales los estudiantes han tenido relación con la investigación, donde muchos de ellos nombraron:

- Los diferentes proyectos de semestre realizados en el ciclo de fundamentación (de primero a sexto semestre).

- El proyecto de quinto semestre donde se proponen diseños experimentales frente a un patrón de vida.

- La práctica pedagógica.

- Los anteproyectos de trabajos de grado.

- Los trabajos de grado.

- Monitorias de investigación.

- El seminario de investigación. 
Memorias del VII Encuentro Nacional de Experiencias en la Enseñanza de la Biología y la Educación Ambiental y II Congreso Nacional de Investigación en la Enseñanza de la Biología

- Actividad propuesta en el espacio de Diversidad Biológica (Tuercas Tornillos).

- Los componentes de Antropología, Enseñanza de la Biología en Colombia, Ecología de poblaciones, Conservación e Interculturalidad.

- Seminarios con las diferentes líneas de Investigación en el PCLB.

- Espacios ajenos a la universidad, es decir en otros contextos.

Igualmente, se logra evidenciar que varias de las personas no responden a este cuestionamiento.

3. Finalidades y problemas abordados en las investigaciones (aproximaciones a investigaciones realizadas)

En esta categoría se evidencia que las problemáticas abordadas corresponden al nivel pedagógico (educativo), como al disciplinar (Biológico). En cuanto al campo disciplinar, encontramos las siguientes temáticas o problemas.

- Diseños experimentales para la enseñanza de un patrón biológico.

- Conceptos en Biología.

- Diversidad biológica.

- Paleobiología.

- Crecimiento y desarrollo de la plenaria

- Irritabilidad en ranas.

- Procesos Biotecnológicos.

- Interacciones ecosistémicas.

- Conservación de especies nacionales en peligro de extinción.

Por otra parte, en cuanto al campo pedagógico y didáctico, encontramos las diferentes herramientas y estrategias utilizadas para realizar los diferentes proyectos como las diferentes estrategias didácticas, las unidades didácticas, los clubes de ciencias, los diseños experimentales, etc. En muchas de las ocasiones estas estrategias fueron utilizadas para abordar temáticas biológicas, pero en otros casos para abordar problemas sociales en los diferentes contextos, en muchos casos a nivel de interculturalidad, como se evidencia en las siguientes unidades de información:

"las temáticas abordadas han estado relacionadas con la enseñanza de la biología en contextos particulares, así como elementos didácticos a través de la propuesta de una unidad didáctica" $(20,2013)$ 
Memorias del VII Encuentro Nacional de Experiencias en la Enseñanza de la Biología y la Educación Ambiental y II Congreso Nacional de Investigación en la Enseñanza de la Biología

"Algunos temas abordados. Diversidad de aprendizaje en el club de Ciencias del Colegio Fals Borda, donde se encontró que por ser un club de Ciencias acepta todos los cursos del colegio van a haber diferencias en el aprendizaje, pero aún así sí aprenden en el club sin ninguna excepción" (18, 2013)

En esta categoría, también se encuentran algunas personas no respondieron a la pregunta.

\section{Relación de la experiencia investigativa con la formación como futuro profesor de Biología.}

En cuanto a las respuestas a la pregunta de la relación de la experiencia investigativa con la formación como futuro profesor de Biología, la mayoría de los estudiantes responden de manera positiva frente a este proceso, debido a que estar relacionado con la investigación en diferentes campos o desde diferentes proyectos educativos realizados en los semestres cursados en el PCLB, les ha permitido comprender aspectos relacionados con el ser y quehacer docente, desde diferentes contextos educativos en los cuales se pueden proponer y desarrollar diferentes proyectos que permitan reconocer la realidad de un contexto educativo específico en la escuela, o en otros campos. Lo anterior se evidencia en las siguientes unidades de información:

"Dichas aproximaciones me han permitido reconocer y comprender aspectos relacionados con el ser y quehacer docente, pues, me he relacionado con contextos educativos en los cuales se proponen y desarrollan proyectos que permitan reconocer la realidad de ámbito educacional" $(19,2013)$

"al igual al largo del recorrido académico hemos planteado proyectos que han permitido el acercamiento de determinados contextos de acuerdo a la problemática que se han planteado" $(7,2013)$

Igualmente, se evidencian diferentes posturas en cuanto a que la relación con la investigación no ha sido de manera rigurosa, ya que los proyectos que se han realizado en el transcurso de la carrera son proyectos momentáneos que no permiten desarrollar un ámbito investigativo en un futuro profesor de Biología.

En esta categoría, al igual que en las anteriores, hay varias personas que no responden al cuestionamiento planteado.

5. ¿Qué ha obstaculizado su relación con la investigación durante su proceso de investigación? 
Memorias del VII Encuentro Nacional de Experiencias en la Enseñanza de la Biología y la Educación Ambiental y II Congreso Nacional de Investigación en la Enseñanza de la Biología

A partir de ésta categoría, se evidencia que la mayoría de las personas muestran su inconformidad frente a la no continuidad de los proyectos que se realizan semestre por semestre, puesto que en todos los semestres del ciclo de fundamentación se realiza un proyecto nuevo para cada eje, donde estos proyectos se quedan en el planteamiento y en muchas ocasiones no son desarrollados. Otro de los obstáculos evidenciados, consiste en que muchas de las temáticas que los estudiantes proponen no se pueden llevar a cabo debido a que estas no se ajustan a las líneas de investigación, haciendo que el estudiante se vea forzado en cambiar sus propuestas, por ejemplo:

"Sin embargo hace falta una linealidad en todos los proyectos, es decir que no sean proyectos inconclusos, si no por el contrario aportaran ejercicios más consistentes en los maestros de formación." $(1,2013)$

"a la hora de presentar el anteproyecto a cualquier Línea de investigación, lo replantean o se modifica de acuerdo a I que requiera la Línea, es decir que si uno no se acerca a la línea antes de pasar el anteproyecto, este no tendrá mucha validez o será replanteada de nuevo" (18, 2013)

Por otra parte, se encuentran obstáculos en los estudiantes a la hora de investigar, entre ellos la falta de docentes y estabilidad que le dan a estos en la universidad para que puedan dirigir los diferentes trabajos investigativos que se proponen. Por último, algunos de los espacios académicos en donde se proponen diferentes actividades académicas, se realizan proyectos solo por cumplir una responsabilidad y no por llevar a cabo los intereses que presentan los estudiantes, como se pone de manifiesto en la siguiente unidad de información:

"por lo general los proyectos se desarrollan para cumplir lo propuesto para la aprobación del semestre, se basa en cumplir con los propósitos de educación" (3, 2013)

Además de las categorías planteadas a partir de las preguntas del instrumento, se plantean dos categorías más, que surgen de las respuestas y de las unidades de información. Obteniendo así categorías referentes a propuestas realizadas por algunos estudiantes, y al interrogante ¿Qué es investigación?

\section{Propuestas}

Algunos estudiantes, al manifestar obstáculos durante su acercamiento o experiencia con la investigación, presentan propuestas para que este proceso sea explícito en el transcurso de la formación docente. Una de las propuestas 
Bio-grafía Escritos sobre la Biología y su Enseñanza.

Edición Extra-Ordinaria. ISSN 2027-1034 P.p $822-837$

Memorias del VII Encuentro Nacional de Experiencias en la Enseñanza de la

Biología y la Educación Ambiental y II Congreso Nacional de Investigación en la Enseñanza de la Biología

hace alusión a la posibilidad de que los proyectos que se realizan en cada semestre tengan continuidad, y que éstos no se queden solo en el planteamiento sino que puedan ser desarrollados.

Para algunas personas es importante aclarar qué es investigación y cómo se desarrolla en el PCLB, puesto que para la mayoría es algo confuso y se está viendo desde otras perspectivas, como por ejemplo la aplicación de entrevistas y encuestas, así se evidencia en las siguientes propuestas:

"se hace un inicio de un nuevo planteamiento en cada semestre entonces esos proyectos se deberían tener una continuidad para sí lograr la profundización de cada tema." $(7,2013)$

"se requiere como proyecto de Licenciatura se establezca a que denominamos investigación en educación si solamente se reduce a hacer encuestas y mostrar tendencias o de verdad estamos aportando para el cambio. Más allá de las raíces metodológicas e instrumentos europeos que frecuentemente adoptamos para «investigar» debemos reconocer nuestro campo de conocimiento y estudio y establecer en este sentido una investigación que sea pertinente para nuestro contexto" $(8,2013)$

\section{7. ¿Qué es investigación?}

Finalmente, en cuanto a esta categoría que emergió de las unidades de información, se encuentran las siguientes apreciaciones tomadas literalmente del instrumento de indagación:

"La investigación es un proceso que se ha de realizar de forma paulatina y con un seguimiento que aporte aprendizaje, datos y permita una indagación mucho más profunda sobre determinado proyecto." $(7,2013)$

"Investigar no es solo preguntarse por lo que acontece, es una transformación sobre el sujeto y el saber, permite leernos a través de múltiples formas y posibilidades. Es también y ha constituido un reto desde la escritura, que me ha permitido verla como un encuentro singular sobre lo que soy como maestra" $(20,2013)$

"La investigación posibilita no solo problematizar sino aportar con algo a dicho aspecto, desde el conocimiento hasta las soluciones concretas" $(11,2013)$

"la investigación no posee un concepto claro para los estudiantes y en muchas veces tampoco para los profesores, se pretende hacer tanto que se termina haciendo poco o nada" $(8,2013)$

"Que no se tiene un concepto claro de investigación, y dichos trabajos no son rigurosos puesto que no se cumple a cabalidad el método de investigación y los métodos de investigación son supremamente cortos, puesto que desde mi punto de vista no se podría llamar investigación"

$(4,2013)$ 
Memorias del VII Encuentro Nacional de Experiencias en la Enseñanza de la Biología y la Educación Ambiental y II Congreso Nacional de Investigación en la Enseñanza de la Biología

De acuerdo a las definiciones anteriormente expuestas, se evidencia que algunos de los estudiantes consideran la investigación como un proceso, el cual se desarrolla paulatinamente a partir del seguimiento de la temática estudiada. Dicho proceso deberá permitir la problematización y la transformación sobre el sujeto y el saber. Para otros estudiantes se evidencia que el referente sobre investigación es subjetivo, en la medida en que ésta se desarrolla dependiendo los procesos e intereses personales.

\section{Conclusiones}

A partir de la aplicación del cuestionario, se aportaron elementos para la caracterización y comprensión de la formación en investigación en futuros profesores del PCLB de la UPN. De acuerdo a los hallazgos obtenidos, es claro que la mayoría de los estudiantes no presenta una perspectiva definida acerca de qué es investigación, ello se evidencia en que las respuestas sobre el desarrollo o acercamiento a procesos investigativos se encuentran divididas, dado que algunos estudiantes manifiestan que no han tenido ningún acercamiento a la investigación durante su proceso de formación en el Departamento de Biología. Por el contrario, para otros estudiantes no se ha desarrollado investigación, pero sí han sido visibles algunos elementos para realizar una investigación, éstos han sido explícitos en los semestres del ciclo de fundamentación del PCLB (de primer a sexto semestre), en actividades desarrolladas en espacios académicos, durante la práctica pedagógica y a partir del planteamiento del anteproyecto de práctica.

Finalmente, mediante el instrumento de evidencia que es importante indagar sobre el proceso de formación en investigación toda vez que muestra cómo los estudiantes se han posicionado frente a las diferentes actividades y/o espacios propuestos por el PCLB para el acercamiento y desarrollo de investigación.

\section{Bibliografía}

Bardín, L. (1977). Analyse de contenu. Paris: Presses Universitaries de France. (Tra. cast. Análisis del contenido. Madrid: Akal, 1986).

Bondarenko, N. (2009) El Componente Investigativo y la Formación Docente en Venezuela. Revista electrónica Estudios pedagógicos, Valdivia, v. 35, n. 1, Disponible en <http://www.scielo.cl/scielo.php?script=sci_arttext\&pid=S071807052009000100015\&lng=es\&nrm=iso>.Accedido en 24 mayo 2013.

Carrasco, J. y Caldero, J. (2000). Aprendo a Investigar en Educación. Madrid: RIALP. 
Bio-grafía Escritos sobre la Biología y su Enseñanza.

Edición Extra-Ordinaria. ISSN 2027-1034 P. p $822-837$

Memorias del VII Encuentro Nacional de Experiencias en la Enseñanza de la Biología y la Educación Ambiental y II Congreso Nacional de Investigación en la Enseñanza de la Biología

Departamento de Biología (1998). Proyecto Curricular Licenciatura en Biología. Bogotá: Universidad Pedagógica Nacional.

Escobar, G. (2012) Formación Investigativa en Futuras Profesoras. El Caso de Dos Estudiantes en el Contexto de la Práctica Profesional Inicial en un Programa de Licenciatura en Biología. Universidad Pedagógica Nacional (Colombia). Tesis para optar el título de Magister en Educación.

Korman (1986). The Focus Group Sensign. New York.

Porlán, R. y Rivero, A. (1998). El conocimiento de los profesores. Sevilla: Díada.

Roa, R. (2011). El control de la profesión docente: algunos apuntes y análisis. Revista Nodos y Nudos. 3 (31), 46-55.

Valbuena E, Castro A, Hernández A. Vallejo C. I (2013) Formación en investigación en futuros profesores de Biología. Proyecto de Investigación. CIUP

Valeiras, N. (2005) La investigación en la formación docente. Memorias de las $\checkmark$ Jornadas Nacionales de Enseñanza de la Biología, Asociación argentina de docentes de Biología, pp 95-97. 